\title{
A CRITERION FOR THE BOUNDEDNESS OF SINGULAR INTEGRALS ON HYPERSURFACES
}

\author{
STEPHEN W. SEMMES
}

\begin{abstract}
This paper gives geometric conditions on a hypersurface in $\mathbf{R}^{n}$ so that certain singular integrals on that hypersurface define bounded operators on $L^{2}$. These singular integrals include the Cauchy integral operator in the sense of Clifford analysis and in particular the double layer potential. For curves in the plane, this condition is more general than the chord-arc condition but less general than the Ahlfors-David condition. The main tool is the $T(b)$ theorem [DJS].
\end{abstract}

\section{INTRODUCTION}

We want to find conditions on $d$-dimensional surfaces $\mathscr{S}$ in $\mathbf{R}^{d+1}$ so that singular integrals of the form

$$
T f(x)=\mathrm{PV} \int_{\mathscr{S}} k(x-y) f(y) d y \quad(x \in \mathscr{S})
$$

are bounded on $L^{p}(\mathscr{S})$, where $d y$ denotes surface measure on $\mathscr{S}$. Here $k(x)$ should be homogeneous of degree $-d$, odd, and smooth away from the origin. The special case of $k(x)=x_{i} /|x|^{d+1}$ arises in connection with the double layer potential and the Cauchy kernel associated to Clifford analysis.

For simplicity, we assume throughout this paper that $\mathscr{S}$ is smooth, even that $\mathscr{S} \cup\{\infty\}$ is a connected smooth submanifold of $S^{d+1}=\mathbf{R}^{d+1} \cup\{\infty\}$, and that $\mathscr{S}$ is orientable, so that there is a smooth, nowhere vanishing normal vector field $n=n_{\mathscr{S}}$ on $\mathscr{S}$. Apparently, algebraic topology implies that $\mathbf{R}^{d+1} \backslash \mathscr{S}$ has exactly two components. (In fact, it also implies that orientability follows from the other assumptions.) However, we are not making any other topological assumptions on $\mathscr{S}$. We do not require that $\mathscr{S}$ be homeomorphic to $\mathbf{R}^{d}$; for example, $\mathscr{S}$ is allowed to have handles. Of course, the estimates we get will not depend on these a priori smoothness assumptions.

Analogous to the $d=1$ situation, we shall assume that for some $K>0$ and all $x \in \mathscr{S}, R>0$ we have

$$
K^{-1} R^{d} \leq\left|B_{R}(x) \cap \mathscr{S}\right| \leq K R^{d} .
$$

Received by the editors August 14, 1987.

1980 Mathematics Subject Classification (1985 Revision). Primary 42B20.

The author was partially supported by the National Science Foundation. 
Here $B_{R}(x)=\{y:|x-y|<R\}$, and $|E|$ denotes the surface measure of $E \subseteq \mathscr{S}$. When $d=1$ the lower estimate in (1.1) is automatic, but when $d>1$ it is a restriction.

This assumption implies that $\mathscr{S}$, equipped with surface measure and the Euclidean distance, is a space of homogeneous type, in the sense of Coifman and Weiss. Thus the usual results of Calderon-Zygmund theory are present; e.g., if $T$ in $(1.1)$ is bounded on $L^{p}(\mathscr{S})$ for some $p$, then it is bounded for all $p, 1<p<\infty$, takes $L^{\infty}$ into $\mathrm{BMO}$, etc.

It is a well-known result of David [D1] that, when $d=1,(1.2)$ is sufficient for the boundedness of $T$ in (1.1) and also necessary if we take $T$ to be the Cauchy integral, for example. David has also observed that (1.2) is not sufficient when $d>1$. We shall give an extra condition that does the job.

Theorem 1. Assume that $\mathscr{S}$ satisfies the a priori assumptions above and also (1.2). Assume also that $\mathscr{S}$ satisfies the following condition:

there is an $A>0$ so that for each $x \in \mathscr{S}$ and $R>0$ we can find $y, z \in \mathbf{R}^{d+1} \backslash \mathscr{S}$ such that $y, z$ live in different components of $\mathbf{R}^{d+1} \backslash \mathscr{S},|y-x|+|z-x| \leq A R$, and $B_{A^{-1} R}(y)$ and $B_{A^{-1} R}(z)$ are disjoint from $\mathscr{S}$.

Then there is a $C=C(A, K, d)$ so that if $P(x)$ is an odd polynomial, homogeneous of degree $l$, and $k(x)=P(x) /|x|^{d+l}$, then the operator $T$ in (1.1) is bounded on $L^{2}(\mathscr{S})$ with norm at most $C^{l}\|P\|_{L^{\infty}\left(S^{d}\right)}$.

Thus, if $K(x)$ is odd, homogeneous of degree $-d$, and real-analytic away from 0 with good enough radius of convergence on $S^{d}$ (how good depending on $A, K$, and $d$ ), then $T$ in (1.1) is bounded. Presumably $k(x)$ should only have to be smooth away from 0 , but the proof will not give this.

Another condition on a surface $\mathscr{S}$ for singular integrals to be bounded on $\mathscr{S}$ has been given by David [D2]. His condition and the one above are quite different, and neither implies the other. When $d=1$, his condition reduces to (1.2) (and his earlier result), which is more general than the theorem above. Theorem 1 does imply the boundedness of the Cauchy integral on chord-arc curves and would even allow the curves to smash into themselves in a "nontangential" fashion. Long thin strips, or tubes, are bad for Theorem 1.

When $d>1$, the two conditions are harder to compare. David's condition is better at letting the surface smash into itself, or cross itself, as in dimension 1. On the other hand, his condition is in terms of a parametrization satisfying certain estimates, while the condition above is more intrinsic. For curves, one can always take the arclength parametrization, while for surfaces one cannot in general find a nice parametrization.

Condition (1.3) holds if one assumes that there is a homeomorphism $h$ of $\mathbf{R}^{d}$ onto $\mathscr{S}$ that is bilipschitz or even just quasisymmetric. This fact, due to Väisälä [V], is proved using a compactness argument. (Of course, in the bilipschitz case 
one could control singular integrals using the method of rotations and the results in $[\mathrm{CDM}]$.)

Condition (1.3) is natural geometrically from the point of view of harmonic analysis. It says that $\mathbf{R}^{d+1}$ and $\mathscr{S} \times \mathbf{R}$ are similar in the sense that $\mathscr{S}^{c}$ has Whitney cubes of roughly the same size in roughly the same places in each of them. Of course, this correspondence of Whitney cubes is not as strong as it is when there is a global quasiconformal mapping on $\mathbf{R}^{d+1}$ that maps $\mathbf{R}^{d}$ onto $\mathscr{S}$.

We shall prove Theorem 1 using the $T(b)$ theorem [DJS]. There is a problem, though, and we must generalize the $T(b)$ theorem, which we do in $\S 3$. Modulo this, the theorem is proved in $\S 2$.

The use of the $T(b)$ theorem in this context is both natural and frustrating. The $T(b)$ theorem permits one to estimate singular integrals without first obtaining an intimate knowledge of the geometry of the surface. It would be very interesting to have a proof of Theorem 1 in the spirit of the good- $\lambda$ arguments of David [D1, D2], or at least something more geometric, but it is not at all clear how.

It would also be interesting to prove harmonic measure estimates in this context. For example, suppose that $D \subseteq \mathbf{R}^{d+1}$ is an NTA domain, in the sense of Jerison and Kenig [JK], and that $\partial D$ satisfies the assumptions of Theorem 1. Is it true that harmonic measure is $A_{\infty}$ equivalent to surface measure when properly localized? When $d=1$, this reduces to Lavrentiev's theorem. If $\mathscr{S}$ is the bilipschitz (or quasisymmetric) image of $\mathbf{R}^{d}$, this is true. This problem is closely related to the preceding one. In both cases you would like somehow to reduce to the known results for Lipschitz graphs by good- $\lambda$ type arguments.

Alternatively, one could hope to use Theorem 1 to get harmonic measure estimates, in the same spirit as solving the Dirichlet problem on Lipschitz domains by layer potentials. The idea would be to show that any $f \in \operatorname{BMO}(\mathscr{S})$ could be written as, say, $g+\operatorname{Re} C(h)$, where $g, h \in L^{\infty}(\mathscr{S})$ and $C$ denotes the Clifford-Cauchy integral (discussed later).

A closely related problem is to show that if $F$ is Clifford-analytic in $D$ and is the Cauchy integral of its boundary values, and if $F^{*}$ denotes the nontangential maximal function of $F$, then $\left\|F^{*}\right\|_{L^{1}(\mathscr{P})} \leq C\|F\|_{L^{1}(\mathscr{P})}$. If $\mathscr{S}=\mathbf{R}^{d}$, this is well known. (See Stein and Weiss [SW, Chapter 6].) If $\mathscr{S}$ is a Lipschitz graph with small constant, one can prove this using the standard argument and the fact that the harmonic measure is close to surface measure. The corresponding result for $L^{p}(\mathscr{S}), 1<p<\infty$, instead of $L^{1}$, is not hard to obtain, using the Cauchy integral formula and (1.3). When $d=1$, one can use factorization (obtained via conformal mapping) to reduce from $p=1$ to $p=2$. For general Lipschitz graphs in higher dimensions, this result is not known. The methods of Uchiyama [U] does not seem to apply easily. 


\section{THE PROOF OF THEOREM 1}

It turns out that Clifford algebras and the associated Cauchy kernel provide a useful algebraic setup for proving Theorem 1, so we first review these topics. See [BDS] for details.

Let $\mathscr{C}_{n}(\mathbf{R})$ denote the Clifford algebra over $\mathbf{R}$ with $n$ generators $e_{1}, \ldots, e_{n}$ and an identity $e_{0}$, defined by the relations $e_{i}^{2}=-e_{0}$ and $e_{i} e_{j}=-e_{j} e_{i}, i \neq j$, $i, j>0$. When $n=1$ this gives the complex numbers, while for $n=2$ quaternions come out. In general, you get an algebra with dimension $2^{n}$. If $x \in \operatorname{span}\left\{e_{0}, e_{1}, \ldots, e_{n}\right\}, x=\sum_{i=0}^{n} x_{i} e_{i}$, set $x^{*}=x_{0} e_{0}-\sum_{i=1}^{n} x_{i} e_{i}$. Then $x x^{*}=x^{*} x=\|x\|^{2}$ and in particular

$$
x^{-1}=x^{*} /\|x\|^{2}, \quad\left\|x^{-1}\right\|=\|x\|^{-1} .
$$

Define two differential operators $D_{L}$ and $D_{R}$ on Clifford-valued functions by $D_{L} f=\sum e_{i} \partial f / \partial x_{i}$ and $D_{R} f=\sum\left(\partial f / \partial x_{i}\right) e_{i}$. When $n=1$, these two operators reduce to $\bar{\partial}$. Define $D_{L}^{*}$ and $D_{R}^{*}$ by $D_{L}^{*} f=e_{0} \partial f / \partial x_{0}-\sum_{1}^{n} e_{i} \partial f / \partial x_{i}$ and similarly for $D_{R}^{*}$. Then $D_{L} D_{L}^{*}=D_{L}^{*} D_{L}=\Delta$, and the same for $D_{R}$.

Solutions of $D_{L} f=0 \quad\left(D_{R} f=0\right)$ are called left (right) Clifford analytic. They are harmonic, hence real analytic.

There is an associated Cauchy kernel

$$
E(x)=x^{*} /|x|^{d+1}
$$

except for a multiplicative constant. This is the fundamental solution of $D_{L}$ and $D_{R}$, as one can easily compute. There is also a corresponding Cauchy integral formula. A special case of that formula is the following. Let $D$ be a bounded domain with smooth boundary, and let $n(x)$ denote the outward unit normal. We can view $n(x)$ as an element of $C_{d}(\mathbf{R})$ by identifying $\mathbf{R}^{d+1}$ with $\operatorname{span}\left\{e_{0}, e_{1}, \ldots, e_{d}\right\}$. Then

$$
\begin{array}{rlrl}
\int_{\partial D} E(x-y) n(y) d y=\int_{\partial D} n(y) E(x-y) d y=0 & & \text { if } x \in \mathbf{R}^{d+1} \backslash \bar{D}, \\
& =a & & \text { if } x \in D,
\end{array}
$$

where $a=a(n) \neq 0$ arises because we have been dropping multiplicative constants.

To prove Theorem 1, we wish to use the $T(b)$ theorem with $b=n$ where $n=n_{\mathscr{S}}$ is a smooth choice of unit normal on $\mathscr{S}$. To be able to apply $T(b)$, we need to show that $n$ satisfies a certain noncancellation condition. This is discussed in detail in $\S 3$. By Theorem 3.7, it is enough to show that there is a $C>0$ so that for any given $x \in \mathscr{S}$ and $R>0$ we can find a real-valued function $\eta$ on $\mathscr{S}$ such that $\operatorname{supp} \eta \subseteq B_{C R}(x),\|\eta\|_{\infty} \leq C R^{-d},\|\eta\|_{\text {Lip } 1} \leq$ $C R^{-d-1}$, and $\left|\int_{\mathscr{P}} n \eta d x\right| \geq C^{-1}$.

Fix $x \in \mathscr{S}$ and $R>0$. Let $y, z$ be as in (1.3), and set $\beta(w)=$ $E(w-y)-E(w-z)$. From (2.1) (and a limiting argument) we get that

$$
\left|\int_{\mathscr{F}} \beta(w) n(w) d w\right|=a \text {. }
$$


We cannot take $\eta=\beta$, because $\beta$ is not real valued or compactly supported. However, because of (1.2) and because $\beta$ decays at $\infty$ like $|x|^{-d-1}$, we can cut $\beta$ off far enough out so that $\left|\int_{\mathscr{S}} \beta(w) n(w) d w\right|$ is still at least $a / 2$. Although $\beta$ is not real valued, we can write $\beta=\sum e_{i} \beta_{i}$, so that for some $i,\left|\int \beta_{i} n\right| \geq$ $a / 2(d+1)$. Take $\eta$ to be this $\beta_{i}$ (cut off at $\infty$, of course).

Thus, by Theorem 3.7 we can apply the $T(b)$ theorem with $b=n$. In other words, if we are given a weakly defined linear operator acting on Clifford-valued functions and if $T$ is associated to a standard kernel and is weakly bounded, then $T$ is bounded on $L^{2}(\mathscr{S})$ if $T(n)$ and $T^{t}(n)$ lie in BMO. (See [DJS] for precise definitions.)

Because the Clifford algebras are not commutative, we must be careful about defining $T^{t}(n)$. For clarity of exposition we assume that $T$ is defined by integration against a (Clifford-valued) kernel:

$$
T f(x)=\int_{\mathscr{S}} k(x, y) f(y) d y, \quad x \in \mathscr{S} .
$$

Then we define $T^{t}$ by

$$
T^{t} f(x)=\int_{\mathscr{S}} f(y) k(y, x) d y, \quad x \in \mathscr{S} .
$$

This is not quite the same as the definition of transpose in the matrix-valued case given in [DJS].

In all the examples we shall work with, the kernel of $T$ will satisfy the standard estimates and also $k(x, y)=-k(y, x)$. As in the scalar case, this last property takes care of weak boundedness. Thus we shall only need to compute $T(n)$ and $T^{t}(n)$.

Let us first take $T$ to be the Cauchy integral, i.e.,

$$
T f(x)=\mathrm{PV} \int_{\mathscr{S}} E(x-y) f(y) d y .
$$

From (2.1) we get that $T(n)=0$ and $T^{t}(n)=0$ in the sense of BMO. Thus $T$ is bounded in $L^{2}(\mathbf{R})$.

This implies Theorem 1 in the case where the degree of the polynomial is 1 . We do the general case by induction. We first need a lemma.

Lemma 2.2. Suppose that $k_{0}(x)$ and $k(x)$ are Clifford valued, smooth, odd, and homogeneous of degree $-d$. Let $T$ and $T_{0}$ be the corresponding singular integrals on $\mathscr{S}$ defined by (1.1). Suppose that

$$
D_{L}(k(x))=D_{R}(k(x))=\frac{\partial}{\partial x_{j}} k_{0}(x), \quad x \neq 0,
$$

for some $j, 0 \leq j \leq d$. If $T_{0}$ is bounded on $L^{2}(\mathscr{S})$, then so is $T$.

First recall that if $U$ is a nice domain, $f$ and $g$ are nice Clifford-valued functions on $\bar{U}$, and $n$ is the outward-pointing unit normal on $\partial U$, then

$$
\int_{\partial U} f n g d y=\int_{U}\left\{\left(D_{R} f\right) g+f\left(D_{L} g\right)\right\} d x
$$

(See [BDS, p. 52].) 
Let us use this to compute $T(n)$. Take $U$ to be the component of $\mathbf{R}^{d+1} \backslash \mathscr{S}$ that $n$ does not point into. Formally, we have for $x \in \mathscr{S}$ that

$$
\begin{aligned}
T(n)(x) & =\mathrm{PV} \int_{\mathscr{P}} k(x-y) n(y) d y \\
& =\int_{U} D_{R}(k(x-y)) d y=\int_{U} \frac{\partial}{\partial y_{j}}\left(k_{0}(x-y)\right) d y \\
& =\operatorname{PV} \int_{\mathscr{S}} k_{0}(x-y) n_{j}(y) d y=T_{0}\left(n_{j}\right)(x), \quad n=\sum e_{j} n_{j} .
\end{aligned}
$$

The second to last equality comes from Green's theorem. Since $n_{j} \in L^{\infty}$ and $T_{0}$ is bounded, $T_{0}\left(n_{j}\right) \in \mathrm{BMO}$.

The preceding calculation is not correct; if you do it rigorously, you will pick up $\delta$-functions at the singularity $x$. However, this will only add an $L^{\infty}$ function to the computation of $T(n)$.

Thus $T(n) \in \mathrm{BMO}$, and similarly $T^{t}(n) \in \mathrm{BMO}$. By the $T(B)$ theorem, $T$ is bounded on $L^{2}(\mathscr{S})$, which proves the lemma.

Let us finish the proof of Theorem 1. Assume that Theorem 1 is known for polynomials of degree $<l$ for some $l$, and we want to prove it for degree $=l$. Let $P(x)$ be odd and homogeneous of degree $l$. We may also assume that $P(x)$ is harmonic, by standard facts about spherical harmonics. (See [S, Chapter 3, §3].)

There is a constant $\beta_{l}=\beta_{l}(d)$ such that

$$
\left(P(x) /|x|^{d+l}\right)^{\wedge}=\beta_{l} P(\xi) /|\xi|^{l+1} .
$$

See [S, p. 73]. In Stein's notation, $\beta_{l}=\gamma_{l, 1}$. Do not forget that we are in $\mathbf{R}^{d+1}$, not $\mathbf{R}^{d}$.

As is well known, a consequence of homogeneity is that

$$
P=\frac{1}{l} \sum_{i} x_{i} \frac{\partial}{\partial x_{i}} P=\sum_{i j} x_{i} x_{j} \frac{1}{l(l-1)} \frac{\partial^{2}}{\partial x_{i} \partial x_{1}} P .
$$

Set

$$
P_{i j}=\frac{1}{l(l-1)} \frac{\partial^{2}}{\partial x_{i} \partial x_{j}} P,
$$

so that $P$ is still harmonic but homogeneous of degree $l-2$. Thus

$$
\beta_{l-2}\left(P_{i j}(\xi) /|\xi|^{l-1}\right)^{\vee}=P_{i j}(x) /|x|^{l-2+d} .
$$

Let $T_{i j}$ denote the operator defined on $\mathscr{S}$ via $(1.1)$ and this last kernel. By the induction hypothesis, $T_{i j}$ is bounded on $L^{2}(\mathscr{S})$.

Set $\xi=\sum_{i=0}^{d} \xi_{i} e_{i}, \xi^{*}=\xi_{0} e_{0}-\sum_{i=1}^{d} \xi_{i} e_{i}$, and define $k_{i j}(x)$ by

$$
\beta_{l}\left(\xi_{i} \xi^{*} P_{i j}(\xi) /|\xi|^{l+1}\right)=k_{i j}(x) \text {. }
$$


Thus $k_{i j}(x)$ is Clifford valued, homogeneous of degree $-d$, and odd. The $e_{j}$ component of its Fourier transform is $\beta_{l} \xi_{i} \xi_{j} P_{i j}(\xi)|\xi|^{-l-1}$, so that $P(x) /|x|^{l+d}$ is built up out of pieces of the $k_{i j}$ 's, by construction.

Moreover, for $x \neq 0$,

$$
D_{R}\left(k_{i j}(x)\right)=D_{L}\left(k_{i j}(x)\right)=\frac{\beta_{l}}{\beta_{l-2}} \frac{\partial}{\partial x_{i}}\left(\frac{P_{i j}(x)}{|x|^{l-2+d}}\right) .
$$

Indeed, one can compute the derivatives as distributions by using the Fourier transform. These global distributional derivatives will differ from the derivatives away from the origin only by $\delta$-masses at the origin.

It follows now from Lemma 2.2 and the induction hypothesis that the operators defined by the kernels $k_{i j}$ and (1.1) are bounded on $L^{2}(\mathscr{S})$. From this we get that the operator corresponding to $P(x)$ is bounded. One can check that at each stage we picked up only constants that do not depend on $l$, and hence we get an estimate in $C^{l}$ in Theorem 1.

This completes the proof of Theorem 1.

One can also prove area function estimates for Clifford-analytic functions on $\mathbf{R}^{d+1} \backslash \mathscr{S}$ using the square function estimates from [DJS]. We omit the details.

\section{AN EXTENSION OF THE $T(b)$ THEOREM}

To apply the $T(b)$ theorem, we have to show that $b$ satisfies a nonoscillation condition. In fact, there are two nonoscillation conditions, para-accretivity and pseudo-accretivity. The second works on spaces of homogeneous type, while the first is only known to work on $\mathbf{R}^{n}$. This is the problem. In proving Theorem 1 , we need to use the para-accretive case on our surface $\mathscr{S}$, and the arguments in [DJS] do not go over to this setting. To fix this we shall show that para-accretive implies pseudo-accretive, even on a space of homogeneous type, assuming one is open-minded about the definition of pseudo-accretivity.

Let us be more precise. For convenience we assume that the underlying space is a surface $\mathscr{S}$ as in Theorem 1, although everything we do goes over to spaces of homogeneous type.

A sequence of functions $\left\{p_{k}(x, y)\right\}_{k=-\infty}^{\infty}$ on $\mathscr{S} \times \mathscr{S}$ will be called an approximation to the identity if the following properties hold, for some $C>0$, $\alpha>0$ :

$$
\begin{aligned}
& p_{k}(x, y)=0 \text { if }|x-y| \geq C 2^{-k} ; \\
& \left\|p_{k}\right\|_{\infty} \leq C 2^{k d} ; \\
& \left|p_{k}(x, y)-p_{k}\left(x, y^{\prime}\right)\right| \leq C 2^{k(d+\alpha)}\left|y-y^{\prime}\right|^{\alpha} ; \\
& \left|p_{k}(x, y)-p_{k}\left(x^{\prime}, y\right)\right| \leq C 2^{k(d+\alpha)}\left|x-x^{\prime}\right|^{\alpha} ; \quad \text { and } \\
& \int_{\mathscr{S}} p_{k}(x, y) d y \equiv 1 \equiv \int_{\mathscr{S}} p_{k}(x, y) d x .
\end{aligned}
$$

It is not hard to construct approximations to the identity-see [DJS]. 
A bounded function $b$ on $\mathscr{S}$ is called pseudo-accretive if there is an approximation to the identity $\left\{p_{k}\right\}$ such that $\sup _{k}\left\|\left(P_{k} b\right)^{-1}\right\|_{\infty}<\infty$, where we set

$$
P_{k} b(x)=\int_{\mathscr{S}} p_{k}(x, y) b(y) d y .
$$

We say that $b$ is para-accretive if there are constants $C>0, \alpha>0$ so that for any $x \in \mathscr{S}$ and $R>0$ we can find a function $\eta=\eta_{x, R}$ on $\mathscr{S}$ such that $\operatorname{supp} \eta \subseteq B_{C R}(x),\|\eta\|_{\infty} \leq C R^{-d},\|\eta\|_{\operatorname{Lip} \alpha} \leq C R^{-d-\alpha}$, and

$$
\left|\int_{\mathscr{S}} \eta(y) b(y) d y\right| \geq C^{-1} \text {. }
$$

Clearly, if $b$ is pseudo-accretive, then $b$ is para-accretive. Conversely, it is shown in [DJS] that if $b$ is para-accretive and $\mathscr{S}=R^{d}$ then there is a sequence of functions $p_{k}$ such that $\left\|\left(P_{k} b\right)^{-1}\right\|_{\infty}$ is uniformly bounded and $p_{k}$ satisfies (3.1), (3.2), (3.3), and (3.5), and instead of (3.4) one has that for each fixed $y$, $p_{k}(\cdot, y)$ is constant on dyadic cubes of length $2^{-k}$. This substitute for (2.4) should be thought of as a smoothness condition still. (Observe, for example, that if $Q$ is a cube, then $\nabla \chi_{Q}$ is a perfectly nice measure.) This condition is good enough so that the arguments that work in the pseudo-accretive case still work just as well.

This approach to para-accretivity does not work on a general surface $\mathscr{S}$ (or space of homogeneous type), because there will not be a good replacement for cubes. Pseudo-accretivity does work in general, so one can ask if para-accretivity always implies pseudo-accretivity. In [DJS2] it is shown that this holds on $\mathbf{R}$, but that on $\mathbf{R}^{d}, d \geq 2$, it fails, because of a topological obstruction. The problem is basically that the unit circle is not contractible. To get rid of this, we allow ourselves to work in quaternions instead of complex numbers. This enlarges substantially the set of invertible elements and gets rid of the topological problem. Because of the noncommutativity, however, one must be more careful about the algebra.

For the application in $\S 2$, we need to do this in more generality, for Clifford algebra-valued functions. Let us first go through the definitions above and indicate how they must be changed in the Clifford-valued case or, more generally, the matrix-valued case.

First of all, for an operator with matrix-valued kernel acting on matrix-valued functions, we define the transpose as in $\S 2$.

A sequence of matrix-valued functions $\left\{p_{k}\right\}$ is called an approximation to the identity if it satisfies (3.1)-(3.5) above. In (3.1), " 1 " means the identity.

A bounded matrix-valued function $b$ is pseudo-accretive if there are two matrix-valued approximations to the identity $\left\{p_{k}\right\}$ and $\left\{\hat{p}_{k}\right\}$ such that $\left\|\left(P_{k} b\right)^{-1}\right\|_{\infty}$ and $\left\|\left(\widehat{P}_{k}^{t} b\right)^{-1}\right\|_{\infty}$ are uniformly bounded and $P_{k} b=\hat{P}_{k}^{t} b$ for all $k$. Here the operators $P_{k}$ and $\widehat{P}_{k}$ are defined in the obvious way by integration against the kernels $p_{k}$ and $\widehat{p}_{k}$. (In the commutative case, we take $\widehat{P}_{k}=P_{k}^{t}$.) 
With these definitions, the $T(b)$ theorem for pseudo-accretive matrix-valued functions $b$ is proved exactly as in [DJS]. One defines the operator $S_{k}$ by $S_{k}=\widehat{P}_{k}\left\{\left(P_{k} b\right)^{-1}\right\} P_{k}$, where $\left\{\left(P_{k} b\right)^{-1}\right\}$ means the operator of multiplication by $\left(P_{k} b\right)^{-1}$. Then the kernel of $S_{k}$ satisfies (3.1)-(3.4) and also $S_{k} b \equiv 1 \equiv S_{k}^{t} b$. (This last formula is why we needed both approximations to the identity.)

This point, about needing two approximations to the identity, seems to have been overlooked in [DJS]. In some interesting special cases, such as when $b$ is accretive, i.e., $\operatorname{Re} b \geq \delta>0$, one can take $p_{k}$ to be real-valued and $\widehat{P}_{k}=P_{k}^{t}$, and this issue does not arise.

Theorem 3.7. Suppose $b$ is a bounded function on $\mathscr{S}$ with values in

$$
\operatorname{span}\left\{e_{0}, e_{1}, \ldots, e_{n}\right\} \subseteq \mathscr{C}_{n}\left(\mathbf{R}^{0}\right)
$$

(There need be no relationship between $n$ and the dimension of $\mathscr{S}$.) Suppose that $b$ is para-accretive, which is defined just as above (see (3.6)) for scalarvalued functions, except that we require $\eta$ to be real-valued. Then $b$ is pseudoaccretive when viewed as a $\mathscr{C}_{n+1}(\mathbf{R})$-valued function. In particular, the $T(b)$ theorem holds for this choice of $b$.

Recall that if $x \in \operatorname{span}\left\{e_{0}, e_{1}, \ldots, e_{n}\right\}$, then $\left\|x^{-1}\right\|=\|x\|^{-1}$. This is why we require $b$ to take values there.

To prove Theorem 3.7 we use the argument given in [DJS2, §10] to show that para-accretivity implies pseudo-accretivity on $\mathbf{R}$. Because that argument was never published, we give the details. We must make suitable changes to get rid of the topological problem by permitting values in $\mathscr{C}_{n+1}(\mathbf{R})$, and we must also construct both $P_{k}$ and $\widehat{P}_{k}$. We do this only for $k=0$, the general case being similar. From now on, we assume that $b$ satisfies the hypotheses of Theorem 3.7.

Lemma 3.8. Let $\left\{\varphi_{j}(x, y)\right\}$ be any nonnegative real-valued approximation to the identity on $\mathscr{S}$. Assume $\varphi_{j}(x, y)=0$ if $|x-y| \geq 2^{-j}$ (which we can always do anyway by relabeling). Then there are a large $j_{0}$ and a small $\delta$ such that if $f(x)=\int_{\mathscr{S}} \varphi_{j_{0}}(x, y) b(y) d y$, then for every $x$ there is an $\tilde{x}$ such that $|x-\tilde{x}| \leq 1$ and $|f(x)| \geq \delta>0$. Also, $\|f\|_{\text {Lip } \alpha} \leq C$.

Let $x \in \mathscr{S}$ be given. Let $\eta=\eta_{x, R}$ be as in the definition of paraaccretivity, with $R$ small enough so that $\operatorname{supp} \eta \subseteq B\left(x, \frac{1}{2}\right)$. Setting $\Phi_{j} b(x)=$ $\int \varphi_{j}(x, y) b(y) d y$, we have

$$
\left|\int_{\mathscr{S}} \eta(y)\left(b(y)-\Phi_{j} b(y)\right) d y\right| \leq C 2^{-j \alpha} .
$$

This comes from the Hölder continuity of $j$. If $j$ is large enough,

$$
\left|\int \eta(y) \Phi_{j} b(y) d y\right| \geq \beta>0 \text {, }
$$

by (3.6). The lemma now follows easily. 
If $f$ were not too small anywhere, then we could take $\widehat{P}_{0}=P_{0}=\Phi_{j_{0}}$. Because $f$ may be small (or zero) sometimes, we have to make corrections. The first correction will be of size $\mu, \mu>0$ to be chosen later (small, smaller than 1 or $\delta$ in Lemma 3.8).

Lemma 3.9. Observe that $f$ (as in Lemma 3.8) takes values in

$$
\operatorname{span}\left\{e_{0}, e_{1}, \ldots, e_{n}\right\} \text {. }
$$

We can find a function $g$ on $\mathscr{S}$, valued in $\operatorname{span}\left\{e_{0}, e_{1}, \ldots, e_{n+1}\right\}$, such that $g(x)=f(x)$ if $\|f(x)\| \geq \mu,\|g(x)\|=\mu$ if $\|f(x)\| \leq \mu$, and $g$ satisfies the Hölder condition

$$
\left|g(x)-g\left(x^{\prime}\right)\right| \leq C\left|x-x^{\prime}\right|^{\alpha}, \quad \alpha>0 .
$$

Let $\Sigma_{+}=\Sigma_{+}^{n+1}=\left\{x \in \mathbf{R}^{n+1}:\|x\|=\mu, x=\sum_{i=0}^{n+1} x_{i} e_{i}, x_{n+1} \geq 0\right\}$. There is a nice bilipschitz map $\rho$ of $\Sigma_{+}$onto $\overline{B_{n+1}(0, \mu)}$, the closed ball in $\mathbf{R}^{n+1}$, which agrees with the identity on $\Sigma_{+} \cap\left\{x_{n+1}=0\right\}$. Define $g$ on $\mathscr{S}$ by $g(x)=f(x)$ if $\|f(x)\| \geq \mu, g(x)=\rho^{-1}(f(x))$ if $\|f(x)\| \leq \mu$. This works. (The Hölder condition on $g$ comes from the one on $f$.)

Now set $h=g-f$, so that $\|h\|_{\infty} \leq 2 \mu$ and $\|f+h\| \geq \mu$ everywhere, $h=0$ when $\|f\| \geq \mu$, and $h$ takes values in $\operatorname{span}\left\{e_{0}, e_{1}, \ldots, e_{n+1}\right\}$. Also, the Lip $\alpha$ norm of $h$ is $\leq C$. To define $P_{0}$ and $\widehat{P}_{0}$, we start with $\Phi_{j_{0}}$ and make corrections, the first of which we define now, in terms of $h$.

Let $\left\{\theta_{l}\right\}$ be a partition of unity on $\mathscr{S}$ such that each $\theta_{l}$ satisfies $0 \leq \theta_{l} \leq 1$, $\left\|\theta_{l}\right\|_{\operatorname{Lip}(\alpha)} \leq C \mu^{-\alpha}, \alpha>0, \mu / 2 \leq \operatorname{diam} \operatorname{supp} \theta_{l} \leq \mu$, and $\operatorname{supp} \theta_{l} \subseteq B$ for some ball $B_{l}$ such that the doubles $2 B_{l}$ have bounded overlap, with bound independent of $\mu$. For each $l$, let $x_{l}$ be any point in $\operatorname{supp} \theta_{l}$, and let $\tilde{x}_{l}$ be as in Lemma 3.8.

Our first corrections will be denoted $\rho_{l}(x, y)$ and $\tau_{l}(x, y)$, associated to $P_{0}$ and $\widehat{P}_{0}$, respectively. If $\left\|f\left(x_{l}\right)\right\| \geq \delta / 10$ ( $\delta$ from Lemma 3.8), then we set $\rho_{l} \equiv 0$ and $\tau_{l} \equiv 0$. If $\left\|f\left(x_{l}\right)\right\|<\delta / 10$, we take $a_{l}=f\left(\tilde{x}_{l}\right)-f(x)$, so that $\left\|a_{l}^{-1}\right\| \leq 2 \delta$, and we define $h_{l}=h \theta_{l}$,

$$
\rho_{l}(x, y)=h_{l}(x)\left(1 / a_{l}\right)\left(\varphi_{j_{0}}\left(\tilde{x}_{l}, y\right)-\varphi_{j_{0}}\left(x_{l}, y\right)\right),
$$

and $\tau_{l}(x, y)=\left(\varphi_{j_{0}}\left(\tilde{x}_{l}, y\right)-\varphi_{j_{0}}\left(x_{l}, y\right)\right)\left(1 / a_{l}\right) h_{l}(x)$. Then:

$$
\begin{aligned}
& \int \rho_{l}(x, y) d y=\int \tau_{l}(x, y) d y=0 \text { for all } l ; \\
& \int \rho_{l}(x, y) b(y) d y=\int b(y) \tau_{l}(x, y) d y \text { for all } l,
\end{aligned}
$$

and when $\left\|f\left(x_{l}\right)\right\|<\delta / 10$, this common value is $h_{l}(x)$;

$$
\begin{aligned}
& \rho_{l}(x, y)=\tau_{l}(x, y)=0 \quad \text { if }|x-y| \geq 3 ; \\
& \left\|\rho_{l}\right\|_{\infty}+\left\|\rho_{l}\right\|_{\text {Lip } \alpha} \mu^{\alpha}+\left\|\tau_{l}\right\|_{\infty}+\left\|\tau_{l}\right\|_{\text {Lip } \alpha} \mu^{\alpha} \leq C \mu .
\end{aligned}
$$


(These $\operatorname{Lip} \alpha$ norms are in $(x, y) \in \mathscr{S} \times \mathscr{S}$, jointly.)

Set $r_{0}(x, y)=\varphi_{j_{0}}(x, y)+\sum_{l} \rho_{l}(x, y)$. This $r_{0}(x, y)$ does not have all the properties that we want for $p_{0}(x, y)$, but it has many of them. Unfortunately, $\int r_{0}(x, y) d x$ may not be 1 . However,

$$
\int_{\mathscr{S}} r_{0}(x, y) d y \equiv 1 \text { and }\left(\int_{\mathscr{S}} r_{0}(x, y) b(y) d y\right)^{-1} \text { is bounded. }
$$

The first follows from (3.10).

For the second, we write

$$
\int_{\mathscr{S}} r_{0}(x, y) b(y) d y=f(x)+\sum \int_{\mathscr{S}} \rho_{l}(x, y) b(y) d y .
$$

Fix $x \in \mathscr{S}$. A term in the sum is zero unless $\theta_{l}(x) \neq 0$ and $\left\|f\left(x_{l}\right)\right\|<\delta / 10$. Suppose there is at least one $l$ such that $\theta_{l}(x) \neq 0$ and $\left\|f\left(x_{l}\right)\right\| \geq \delta / 10$. For this $l$ we write $f(x)=f\left(x_{l}\right)+\left(f(x)-f\left(x_{l}\right)\right)$, and because diam supp $\theta_{l} \leq \mu$, we have $\left|x-x_{l}\right| \leq \mu$, so that $\left|f(x)-f\left(x_{l}\right)\right| \leq C \mu^{\alpha}$. If $\mu$ is small enough, this gives $\left\|f(x)^{-1}\right\| \leq C \delta^{-1}$, because $\left\|f\left(x_{l}\right)^{-1}\right\| \leq 10 \delta^{-1}$. Because supp $\theta_{l}$ have bounded overlap, with bound independent of $\mu$, we get

$$
\begin{aligned}
\left|\int_{\mathscr{S}} r_{0}(x, y) b(y) d y-f(x)\right| & \leq \sum_{k}\left|\int_{\mathscr{S}} \rho_{k}(x, y) b(y) d y\right| \\
& \leq C \mu \quad\left(\text { since }\|h\|_{\infty} \leq 2 \mu\right) .
\end{aligned}
$$

Thus if $\mu$ is small enough, we may conclude that $\left\|\left(\int r_{0}(x, y) b(y) d y\right)^{-1}\right\| \leq$ $C \delta^{-1}$.

If there is no $l$ for which $\theta_{l}(x) \neq 0$ and $\left\|f\left(x_{l}\right)\right\| \geq \delta / 10$, then

$$
\int_{\mathscr{S}} r_{0}(x, y) b(y) d y=f(x)+\sum h_{l}(x)=f(x)+h(x)=g(x),
$$

and we know that $\|g(x)\| \geq \mu$ everywhere and $g(x) \in \operatorname{span}\left\{e_{0}, e_{1}, \ldots, e_{n+1}\right\}$, so that $\left\|g(x)^{-1}\right\| \leq \mu^{-1}$.

To get $p_{0}(x, y)$ with all the desired properties, we need to add another correction to $r_{0}(x, y)$ to fix the fact that $\int r_{0}(x, y) d x$ may not be 1 . Note that $\left|\int r_{0}(x, y) d x-1\right| \leq C \mu$, i.e., is small. The idea is to perturb $r_{0}(x, y)$ only where $f(x)$ is big, so as to keep $\int p_{0}(x, y) b(y) d y$ from getting small.

For each $l$, choose a nonnegative bump function $\psi_{l}$ supported in $B\left(\tilde{x}_{l}, \varepsilon\right)$, where $\varepsilon>0$ is very small and will be chosen later, in a way that depends only on $\delta$ and $\|f\|_{\operatorname{Lip} \alpha}$, but not $\mu$. We also require that $\int_{\mathscr{S}} \psi_{l}(x) d x=1$ and $\left\|\psi_{l}\right\|_{\text {Lip } \alpha}$ is bounded, independently of $\mu$.

Define $m_{l}(x, y)$ and $n_{l}(x, y)$ as follows. If $\left\|f\left(x_{l}\right)\right\| \geq \delta / 10$, then $m_{l} \equiv 0$ and $n_{l} \equiv 0$. (Notice that $\rho_{l} \equiv 0$ and $\tau_{l} \equiv 0$ in this case also.) Otherwise, if $\left\|f\left(x_{l}\right)\right\| \geq \delta / 10$, set

$$
m_{l}(x, y)=\left(\int_{\mathcal{P}} h_{l}(x) d x\right) \frac{1}{a_{l}} \psi_{l}(x)\left(\varphi_{j_{0}}\left(\tilde{x}_{l}, y\right)-\varphi_{j_{0}}\left(x_{l}, y\right)\right)
$$


and $n_{l}(x, y)=\psi_{l}(x)\left(\varphi_{j_{0}}\left(\tilde{x}_{l}, y\right)-\varphi_{j_{0}}\left(x_{l}, y\right)\right)\left(1 / a_{l}\right) \int_{\mathscr{S}} h_{l}(x) d x$. Then

$$
\begin{aligned}
& m_{l}(x, y)=n_{l}(x, y)=0 \quad \text { if }|x-y| \geq 3 ; \\
& \left\|m_{l}\right\|_{\infty}+\left\|m_{l}\right\|_{\operatorname{Lip} \alpha}+\left\|n_{l}\right\|_{\infty}+\left\|n_{l}\right\|_{\operatorname{Lip} \alpha} \leq C \mu^{d+1} ; \\
& \int_{\mathscr{S}} m_{l}(x, y) d y=0=\int_{\mathscr{S}} n_{l}(x, y) d y \quad \text { for all } l, x ; \\
& \int_{\mathscr{S}} m_{l}(x, y) d x=\int_{\mathscr{S}} \rho_{l}(x, y) d x \text { and } \\
& \int_{\mathscr{S}} n_{l}(x, y) d x=\int_{\mathscr{S}} \tau_{l}(x, y) d x \text { for all } l, y ; \\
& m_{l}(x, y)=0=n_{l}(x, y) \quad \text { if }\left|x-\tilde{x}_{l}\right| \geq \varepsilon
\end{aligned}
$$

by definition of $\psi_{l}$.

If $\varepsilon>0$ is small enough (depending only on $\delta$ and $\|f\|_{\operatorname{Lip} \alpha}$ ), then $\left|x-\tilde{x}_{l}\right|<$ $\varepsilon$ implies $\|f(x)\| \geq \delta / 3$. Thus we get from (3.20) that

$$
\int_{\mathscr{S}} m_{l}(x, y) b(y) d y=0=\int_{\mathscr{S}} b(y) n_{l}(x, y) d y \quad \text { if }|f(x)|<\frac{\delta}{3} .
$$

Define

$$
p_{0}(x, y)=\varphi_{j_{0}}(x, y)+\sum \rho_{l}(x, y)-\sum m_{l}(x, y)
$$

and

$$
q_{0}(x, y)=\varphi_{j_{0}}(x, y)+\sum \tau_{l}(x, y)-\sum n_{l}(x, y),
$$

and set $\widehat{P}_{0}(x, y)=q_{0}(y, x)$. By construction, $p_{0}$ and $q_{0}$ satisfy conditions (3.1) $-(3.5)$ for $k=0$. Also, by definitions,

$$
\int_{\mathscr{S}} p_{0}(x, y) b(y) d y=\int_{\mathscr{S}} b(y) q_{0}(x, y) d y=\int_{\mathscr{S}} b(y) \widehat{P}_{0}(y, x) d y
$$

for all $x \in \mathscr{S}$. (This was true for the $\rho_{l}$ 's and $\tau_{l}$ 's and for the $m_{l}$ 's and $n_{l}$ 's.)

Set $k(x)=\int_{\mathscr{S}} p_{0}(x, y) b(y) d y$. We must show that $k(x)^{-1}$ is bounded. Fix $x \in \mathscr{S}$. Suppose that $\|f(x)\|<\delta / 3$. By (3.21), $k(x)=\int r_{0}(x, y) b(y) d y$, and we have already seen that this has a bounded inverse (3.14).

Now suppose that $\|f(x)\| \geq \delta / 3$, so that $\left\|f(x)^{-1}\right\| \leq 3 / \delta$. Then

$$
|k(x)-f(x)| \leq \sum_{l}\left|\int_{\mathscr{S}} \rho_{l}(x, y) b(y) d y\right|+\sum_{l}\left|\int_{\mathscr{S}} m_{l}(x, y) b(y) d y\right| .
$$

Because $\left\{\operatorname{supp} \theta_{j}\right\}$ has bounded overlap and $\|h\|_{\infty} \leq C \mu$, we obtain

$$
\sum_{l}\left|\int_{\mathscr{S}} \rho_{l}(x, y) b(y) d y\right| \leq C \mu \text {. }
$$

Also, for a given $x$, there are at most $C \mu^{-d}$ different $l$ 's such that $\varphi_{l}(x) \neq 0$. Hence there are at most $C \mu^{-d}$ different $l$ 's such that $\left|\int m_{l}(x, y) b(y) d y\right| \neq 0$. 
From this, (3.16), and (3.17) we get

$$
\sum_{l}\left|\int_{\mathscr{S}} m_{l}(x, y) b(y) d y\right| \leq C \mu \text {. }
$$

From (3.22) it follows that $\left|k(x)^{-1}\right| \leq 10 / \delta$ if $|f(x)| \geq \delta / 3$ and $\mu$ is small enough.

This completes the construction of $p_{0}(x, y)$ and $\hat{p}_{0}(x, y)$. Theorem 3.7 is now proved.

\section{REFERENCES}

[BDS] F. Brackx, R. Delanghe, and F. Sommer, Clifford analysis, Pitman, 1982.

[CDM] R. Coifman, G. David, and Y. Meyer, La solution des conjectures de Calderón, Adv. in Math. 48 (1983), 144-148.

[D1] G. David, Opérateurs intégraux singuliers sur certains courbes du plan complex, Ann. Sci. École Norm. Sup. 17 (1984), 157-189.

[D2] __ Opérateurs d'intégrale singulière sur les surfaces régulières, Ann. Sci. École Norm. Sup. (to appear).

[DJS1] G. David, J.-L. Journé, and S. Semmes, Opérateurs de Calderón-Zygmund, fonctions paraaccrétives, et interpolation, Rev. Mat. Iberoamer. 1 (1985), 1-56.

[DJS2] _ Calerón-Zygmund operators, para-accretive functions, and interpolation, preprint.

[JK] D. Jerison and C. Kenig, Boundary behaviour of harmonic functions in nontangentially accessible domains. Adv. in Math. 46 (1982), 80-147.

[S] E. M. Stein, Singular integrals and differentiabilty properties of functions, Princeton Univ. Press, 1970.

[SW] E. M. Stein and G. Weiss, Introduction to Fourier analysis on Euclidean spaces, Princeton Univ. Press, 1971.

[U] A. Uchiyama, A constructive proof of the Fefferman-Stein decomposition for $\mathrm{BMO}\left(\mathbf{R}^{n}\right)$, Acta Math. 148 (1982), 215-241.

[V] J. Väisälä, Quasimöbius invariance of uniform holes, preprint.

Department of Mathematics, Yale University, New Haven, Connecticut 06520

Current address: Department of Mathematics, Rice University, Houston, Texas 77251 